\title{
CA 125 is a better marker to differentiate endometrial cancer and abnormal uterine bleeding
}

\author{
Nithin Kumar $\mathrm{U}^{1 \mathrm{a}}$, Sridhar $\mathrm{MG}^{1}$, Srilatha $\mathrm{K}^{1 \mathrm{~b}}$, Habebullah $\mathrm{S}^{2}$
}

1. Department of Biochemistry, 2. Department of Obstetrics and Gynecology, Jawaharlal Institute of Post graduate Medical Education and Research, Puducherry - 605006.

a. Present Address: Department of Biochemistry, Sri Ramachandra Institute of Higher Education and Research, Porur, Chennai - 600116

b. Present Address: Department of Biochemistry, Captain Srinivasa Murthy Regional Ayurveda Drug Development Institute, CCRAS, M/o AYUSH, Arumbakkam, Chennai - 600106.

\begin{abstract}
Background: Incidence of endometrial cancer in India is increasing due to lifestyle changes and obesity. As 5 year survival rate of cancer confined to uterus is good, there is need for serum tumor marker for early diagnosis. This study was designed to identify a tumor marker which differentiate endometrial carcinoma and abnormal uterine bleeding (AUB) because common presentation of endometrial carcinoma is AUB.

Objectives: To estimate and compare serum prolactin, Cancer Antigen 125 (CA-125), Cancer Antigen 15-3 (CA15-3), and Carcino embryonic antigen (CEA) levels in patients with endometrial cancer and abnormal uterine bleeding; To evaluate the role of these markers in diagnosing endometrial cancer.

Methodology: Thirty eight patients with endometrial cancer and 40 patients with AUB were recruited in this study. Serum prolactin, CA 125, CEA, and CA 15-3 levels were estimated in both groups.

Results: The levels of CA 15-3, CA 125, CEA, and prolactin were increased in endometrial carcinoma patients, on comparison with AUB patients. CA 125 alone was found to be a better marker to detect endometrial cancer with 52.63\% sensitivity, $80.00 \%$ specificity.

Conclusion: As individual tumor marker, serum CA 125 has the ability to detect endometrial cancer in patients with abnormal uterine bleeding.

Keywords: CA-125 antigen, CA 15-3, carcino embryonic antigen, endometrial carcinoma, prolactin, tumor marker, abnormal uterine bleeding.

DOI: https://dx.doi.org/10.4314/ahs.v18i4.17

Cite as: Nithin KU, Sridhar MG, Srilatha K, Habebullah S. CA 125 is a better marker to differentiate endometrial cancer and abnormal uterine bleeding. Afri Health Sci. 2018;18(4): 972-978. bttps:/ / dx.doi.org/10.4314/abs.v18i4.17
\end{abstract}

\section{Introduction}

Endometrial cancer is a common gynecological malignancy in women and its prevalence in India is increasing in last 5 years. According to GLOBOCAN 2012 statistics, approximately 12,300 new cases are diagnosed yearly, of which about 4700 women die from the disease each year

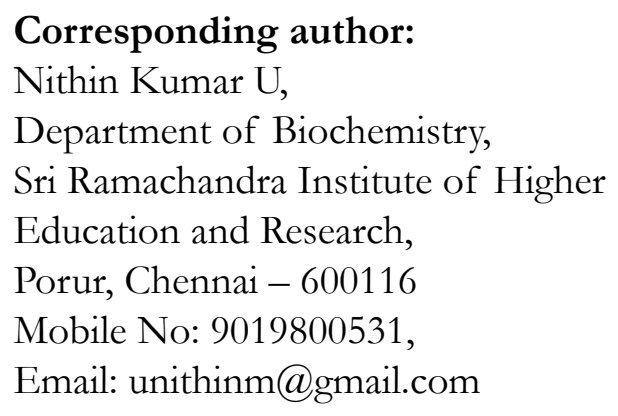

in India ${ }^{1}$. Recently published data showed that the incidence of Endometrial cancer 4.3 per 100000 in India ${ }^{2}$. The overall 5-year survival rate is $86 \%$ when all stages combined and for the disease confined to the uterus is $96 \% 0^{3,4}$. Most common presentation of endometrial cancer is abnormal uterine bleeding. Among them, around $75 \%$ of the women were diagnosed to have early stage of endometrial cancer ${ }^{5}$. AUB being most common presentation in many gynecological diseases and there is a need for markers there is a need for markers to diagnose endometrial carcinoma, in patients with similar symptoms such as AUB.

If endometrial cancer is detected in early stages, treatment can reduce overall mortality. Endometrial cancer is diagnosed either by histo-pathological examination of 
endometrial biopsy specimen or by endometrial cytological examination of endometrial brush. These methods are invasive and, less sensitive in diagnosing endometrial cancer and chances of false negative rates are high ${ }^{6}$. This warrants the use of non-invasive serum markers to detect endometrial cancer.

Earlier studies have estimated serum concentrations of Cancer Antigen -125 (CA 125) and Cancer Antigen 15-3 (CA 15 - 3) in endometrial cancer. These studies have demonstrated an association between preoperative serum CA 125 and CA 15-3 levels with tumor stage and showed that these markers can be used to predict of extra uterine spread of cancer and can be used to monitor the response of chemotherapy as well ${ }^{7,8}$. Elevated CA 15-3 indicate poor prognosis in these patients ${ }^{9}$. A study done by Neunteufel W et al. has found 58\% positivity for Carcino Embryonic Antigen (CEA) in immunohistochemistry studies done in 40 endometrial cancer cases CEA in immunohistochemistry studies done in 40 endometrial cancer cases ${ }^{10}$.

A study by Yurkovetsky Z et al. has demonstrated elevated prolactin levels in endometrial carcinoma and proposed that prolactin has got high diagnostic power in detecting early cancer ${ }^{11}$. Kanat-Pektas $\mathrm{M}$ et al. suggested that CA125 and prolactin are non-specific for endometrial cancer. As Prolactin is subjected to physiological variation, measuring this hormone will have limited use in diagnosing endometrial cancers at initial stage. Hence, it is difficult to use either Prolactin or CA-125 as a single marker to screen endometrial cancer. The same study also showed that these two markers should be included together as a part of biochemical screening panel in future ${ }^{12}$.

The objective of this study includes comparison of tumour markers in two different conditions have same presentation. So the patients with AUB were included as comparison group. The tumour markers level were compared between these two groups to find out whether any of the included tumour markers can be able to differentiate endometrial carcinoma and AUB though there is common clinical presentation.

The present study is undertaken to estimate and compare the concentrations of serum prolactin, CA 125, CA 153 , and CEA in patients with endometrial cancer and abnormal uterine bleeding and also to evaluate whether the above parameters can be used to diagnose endometrial cancer.

\section{Methodology}

This study was conducted in Department of Biochemistry along with Department of Obstetrics and Gynaecology, JIPMER, Puducherry, India. The study was approved by JIPMER institute ethics sub-committee- human studies, and was funded by Intramural grant from JIPMER.

\section{Selection of study participants}

The study subjects were enrolled in the study based on inclusion and exclusion criteria. All perimenopausal women with endometrial carcinoma diagnosed by histopathological examination were taken into group 1 . Similarly weight matched peri-menopausal women with abnormal uterine bleeding other than endometrial carcinomas were considered into group 2. All endometrial cancer patients on treatment and those who were taking hormone replacement therapy were excluded from the study. Patients with abdominal tuberculosis were also excluded as elevated CA 125 levels are associated with this condition ${ }^{13}$.

All patients attending Gynaecology Department, JIPMER Hospital with symptoms of peri-menopausal bleeding during reference period of January 2011 to April 2012 were recruited into study groups as per inclusion and exclusion criteria after obtaining a written informed consent. Thirty eight patients with endometrial carcinoma were included in Group 1, whereas forty weight matched patients with abnormal uterine bleeding other than endometrial cancers were considered into group 2 .

\section{Data collection and Biochemical analyses}

The details of age, height and weight of all participants were recorded and Body Mass Index (BMI) is calculated Body Mass Index BMI is calculated. The height was measured in centimeters using measuring tape and weight was measured in kilogram using Equinox weighing machine (New Delhi, India).

Five $\mathrm{ml}$ of venous blood sample was collected from all study subjects. Serum was separated after centrifugation and stored at $-80^{\circ} \mathrm{C}$ for assays of various parameters as per protocol.

BMI was calculated by using Quetelet's index, [weight / height $\left.{ }^{2}\right]$ The BMI was expressed as $\mathrm{kg} / \mathrm{m}^{2}$. Serum concentrations of CA125, CEA and Prolactin were measured by a two-site sandwich immunoassay using direct chemiluminometric technology (ADVIA Centaur ${ }^{\circledR}$ CP Immunoassay System, Siemens, Switzerland). Whereas CA 15-3

African Health Sciences Vol 18 Issue 4, December, 2018 
was assayed using Enzyme Linked Immuno Sorbent Assay (ELISA) kit Syntron Bioresearch, California ${ }^{17}$. The reference ranges for serum CA-125, Prolactin, and CEA are less than $35 \mathrm{U} / \mathrm{mL}, 1.9-25 \mathrm{ng} / \mathrm{mL}$ and $0-3 \mu \mathrm{g} / \mathrm{L}$ respectively. The reference range for CA $15-3$ is $<33 \mathrm{U} /$ $\mathrm{mL}$.

\section{Statistical analyses}

The normality of the continuous data was checked by Kolmogorov-Smirnov test. Data were reported as mean and standard deviation for normally distributed data and median with interquartile range was used for non-Gaussian data. The levels of tumor makers of two groups were compared either using independent t test or Mann Whitney $\mathrm{U}$ test according to their distribution. The possible relationship among the values of the tumor markers was assessed by correlation analysis. Sensitivity, specificity, positive predictive value and negative predictive value were calculated. Receiver operating Characteristics ROC curve was used to demonstrate the sensitivity of all these markers. The statistical analyses were done at $5 \%$ level of significance and $\mathrm{p}$ value $<0.05$ was considered signifi- cant. The statistical analysis was carried out using Statistical Package for SOcial Sciences (SPSS), Version 20.0

\section{Results}

Thirty eight histo-pathologically confirmed endometrial cancer patients were included first group. Forty weight matched cases of abnormal uterine bleeding other than patients with endometrial cancer were included into group 2.

The demographic characteristics such as age, height, weight and BMI were compared between two groups. There parameters did not differ significantly between study groups. Table 1. The hormonal imbalance in post-menopausal stage can contribute to the change in the tumour markers levels. Therefore, menopausal status also matched between two groups. Details are given in Table 1 . In study group 1, endometrioid adenocarcinoma In study group 1, endometrioid adenocarcinoma of uterus accounted for $36(95 \%)$ of subjects on histopathological analysis. One case each of uterine papillary serous carcinoma and poorly differentiated carcinoma were included in the study.

Table 1: Comparison of demographic characteristics among patients with endometrial cancer and abnormal uterine bleeding

\begin{tabular}{lccc}
\hline Variables & $\begin{array}{c}\text { Endometrial } \\
\text { cancer }(\mathbf{n}=\mathbf{3 8})\end{array}$ & $\begin{array}{c}\text { Abnormal } \\
\text { uterine } \\
\text { bleeding } \\
(\mathbf{n}=\mathbf{4 0})\end{array}$ & p value \\
\hline Age (years) & $49.8 \pm 7.2$ & $48.3 \pm 4.5$ & NS \\
Height (cm) & $151.3 \pm 5.5$ & $152.7 \pm 4.5$ & NS \\
Weight (kg) & $60.2 \pm 11.5$ & $59.4 \pm 12.6$ & NS \\
BMI (kg/m $\left.{ }^{2}\right)$ & $26.4 \pm 5.7$ & $25.4 \pm 5.1$ & NS \\
Menopausal status* & $13 / 25$ & $14 / 26$ & NS \\
(Pre / Post) & & & \\
\hline
\end{tabular}

Data are expressed as Mean $\pm \mathrm{SD}, *$ indicates number of women in each group; NS- not significant

Among the tumor markers, serum levels of CA15-3, CA 125, CEA and prolactin were significantly high in patients with carcinoma than AUB patients (Table 2). than AUB patients Table 2 . We carried out correlation analysis be- tween/ among the different parameters in study group consisting of endometrial carcinoma cases. No significant correlation was observed between CA 125, CA 15-3, CEA, and Prolactin. 
Table 2: Comparison of CA 15-3, CA 125, CEA, and prolactin among patients with endometrial cancer and patients with abnormal uterine bleeding

\begin{tabular}{|c|c|c|c|}
\hline Parameters & $\begin{array}{l}\text { Endometrial } \\
\text { Cancer } \\
(\mathbf{n}=\mathbf{3 8})\end{array}$ & $\begin{array}{l}\text { Abnormal } \\
\text { uterine bleeding } \\
(n=40)\end{array}$ & p value \\
\hline CA $15-3(\mathrm{U} / \mathrm{mL})$ & $\begin{array}{l}21.97 \\
(12.53-48.51)\end{array}$ & $\begin{array}{l}18.75 \\
(9.38-23.89)\end{array}$ & $0.048 *$ \\
\hline CA $125(\mathrm{U} / \mathrm{mL})$ & $\begin{array}{l}19.85 \\
(7.2-47.78)\end{array}$ & $\begin{array}{l}10.95 \\
(6.73-16.97)\end{array}$ & $0.015 *$ \\
\hline CEA $(\mu \mathrm{g} / \mathrm{L})$ & $\begin{array}{l}2.30 \\
(1.58-5.03)\end{array}$ & $\begin{array}{l}2.00 \\
(1.45-2.40)\end{array}$ & $0.033 *$ \\
\hline Prolactin (ng/mL) & $\begin{array}{l}8.36 \\
(6.2-14.3)\end{array}$ & $\begin{array}{l}7.02 \\
(4.93-10.02)\end{array}$ & $0.042 *$ \\
\hline
\end{tabular}

Data are expressed as median (IQR) IQR - Inter-quartile range; *-Statistically significant

ROC curves were plotted for serum levels of CA 125, CA 15 -3, CEA and Prolactin to obtain optimal cut off values to distinguish between endometrial cancer and abnormal uterine bleeding. The ROC curve for CA 15-3 showed an area under curve AUC of 0.630 . The sensitivity and specificity for the cut off of $27.55 \mathrm{U} / \mathrm{mL}$ were $44.74 \%$ and $82.5 \%$ respectively. Positive predictive value PPV of $70.8 \%$ and Negative predictive value NPV of $61.1 \%$ were obtained with the same cut off.

AUC of 0.660 was obtained in ROC curve for CA-125. A cut off of $17.8 \mathrm{U} / \mathrm{mL}$ showed a sensitivity and spec- ificity of $52.63 \%$ and $80 \%$ respectively. PPV of $71.4 \%$ and NPV of $64 \%$ were obtained with the same cut off. The ROC curve for CEA showed an AUC of 0.628. The sensitivity and specificity for the cutoff of $3.6 \mu \mathrm{g} / \mathrm{L}$ were $34.21 \%$ and $95 \%$ respectively. A PPV of $86.7 \%$ and NPV of $60.3 \%$ were obtained with the same cut off. Area under curve of 0.634 was obtained for ROC curve for Prolactin. A cut off of $11.27 \mathrm{ng} / \mathrm{mL}$ showed a sensitivity and specificity of $38.64 \%$ and $87.5 \%$ respectively. PPV of $73.68 \%$ and NPV of $59.3 \%$ were obtained with the same cut off value (Table 3).cut off value Table 3. All tumor markers in our study had a very good specificity, but very less sensitivity at the cut off when taken individually.

Table 3: Sensitivity, specificity, PPV and NPV for tumor markers in endometrial cancer

\begin{tabular}{lllllll}
\hline Parameter & AUC & Cut off value & Sensitivity & Specificity & PPV & NPV \\
\hline CA15-3 & 0.630 & $27.55 \mathrm{U} / \mathrm{mL}$ & $44.74 \%$ & $82.50 \%$ & $70.8 \%$ & $61.1 \%$ \\
CA125 & 0.660 & $17.8 \mathrm{U} / \mathrm{mL}$ & $52.63 \%$ & $80.00 \%$ & $71.4 \%$ & $64 \%$ \\
CEA & 0.628 & $3.6 \mu \mathrm{g} / \mathrm{L}$ & $34.21 \%$ & $95.00 \%$ & $86.7 \%$ & $60.3 \%$ \\
Prolactin & 0.634 & $11.27 \mathrm{ng} / \mathrm{mL}$ & $38.64 \%$ & $87.5 \%$ & $73.7 \%$ & $59.3 \%$ \\
\hline
\end{tabular}

AUC - area under curve for ROC, PPV - Positive predictive value, NPV - Negative predictive value 


\section{Discussion}

Endometrial cancer is a challenging gynecological disease, where a noninvasive screening method is not available. In this study, we estimated serum concentrations of CA 125, CA 15-3, CEA and prolactin and their utility in diagnosis of endometrial carcinoma. There are contradictory reports regarding CA 15-3 levels in endometrial carcinoma patients. Scambia et al and Panici et al have reported an increase in the levels of CA 15-3 in carcinoma cases when compared with endometrial hyperplasia patients ${ }^{8,9}$. In our study, CA 15-3 levels are significantly increased in carcinoma patients in comparison with patients with uterine bleeding. Both these studies are limited by lack of control group. In contrast to these studies Kanat-Pektas et al observed low values of CA 15-3 in patients with endometrial cancer patients when compared to controls ${ }^{12}$. The increase in CA 15-3 observed in our study might be because of increased shedding of cancer antigens to the blood stream.

Erbagci et al studied the variation in levels of CA-125, CA 19-9, CA 15-3, AFP and CEA with menstrual cycle in small number of healthy women. The blood samples were collected between $7^{\text {th }}$ and $21^{\text {st }}$ day of menstrual cycle. The mid luteal phase concentrations of CA 15-3 were significantly higher than mid follicular phase values. There is no significant difference in the level of other tumour markers including CA 125 . In our study most of the women came to the hospital with the complaints of prolonged bleeding. Therefore phase of menstrual cycle cannot be predicted since they have prolonged menstrual phase $^{18}$.

Even though several studies have reported the importance of CA 125 in the diagnosis of ovarian tumor, only limited studies have assessed its role in endometrial cancer. In our study the levels of CA 125 are higher in endometrial cancer patients when compared with abnormal uterine bleeding patients. These findings are in accordance with previous studies and the elevation of CA 125 is due to the increased synthesis of this cancer antigen by endometrial tumor cells $\mathrm{s}^{12,19,20}$.

Jiang $\mathrm{T}$ et al studied importance of preoperative CA 125 levels, age menopausal status and tumor histology in a large number of 995 endometrial cancer patients. The study showed that CA 125 levels had significant association with age and menopausal status, but it was not

African Health Sciences Vol 18 Issue 4, December, 2018 associated with tumour histology. They have suggested different cut-off values for prediction of lymph node metastasis and adnexal involvement and concluded that preoperative CA 125 levels has to be involved in the decision of management ${ }^{21}$.

CA 125 levels are elevated in benign physiological and pathological conditions including menstrual cycle, pregnancy, endometriosis and also in malignant conditions such as endometrial carcinoma ${ }^{22}$. In order to differentiate those conditions from malignancy, patients with abnormal uterine bleeding due to non-malignant cause were included in this study for comparison. The median value of CA-125 is significantly higher $19.85 \mathrm{U} / \mathrm{ml}$ in patients with endometrial carcinoma than patients with AUB $10.95 \mathrm{U} /$ $\mathrm{ml}$. This shows that CA-125 can differentiate malignancy from non-malignant endometrial conditions, though all patients presented with similar symptoms. A study by Povolotskaya $\mathrm{N}$ et al revealed a preoperative CA 125 level greater than $28 \mathrm{U} / \mathrm{ml}$ had significantly correlation with patient's age, tumour grade and unfavorable histological type in 98 uterine cancer patients. They concluded that CA 125 is an inexpensive and reproducible tumour marker providing information about the metastasis and prognosis in endometrial cancer ${ }^{23}$.

CEA is a non-specific tumor marker for gastrointestinal tract tumor and their alteration is not reported much in endometrial cancer. Previous study by Kanat-Pektas et al did not observe any difference in CEA levels between endometrial cancer patients and healthy controls ${ }^{12}$. In current study CEA levels are significantly higher in endometrial cancer cases in comparison with women with abnormal uterine bleeding. CEA is well known marker of colorectal cancer. However, elevated levels of CEA are reported in many carcinomas of epithelial origin like, lung adenocarcinoma, ovarian carcinoma and endometrial adenocarcinoma ${ }^{24}$. Kozakiewicz B et al showed that elevated CEA levels were not sensitive and specific enough to diagnose and monitor treatment of endometrial can$\operatorname{cer}^{25}$.

Prolactin has been proposed as one of biomarkers in endometrial cancer and previous investigators have documented an increase in prolactin levels in endometrial carcinoma cases ${ }^{11,12}$. We observed significantly high serum levels of prolactin in cancer patients when compared to abnormal uterine bleeding group. The higher levels of prolactin may be explained by excessive prolactin release from the endometrial tumor cells ${ }^{12}$. 


\section{Diagnostic utility of serum tumor markers in endo- metrial carcinoma}

The cut offs provided by ROC curve analysis were used to distinguish endometrial cancer patients and AUB cases. The cutoff of $27.55 \mathrm{U} / \mathrm{mL}$ for CA 15-3 showed sensitivity, specificity, PPV and NPV of $44.74 \%, 82.5 \%$, $70.8 \%$ and $61.1 \%$ respectively. A cut off of $17.8 \mathrm{U} / \mathrm{mL}$ for CA 125 showed sensitivity, specificity, PPV and NPV of $52.63 \%, 80 \%, 71.4 \%$ and $64 \%$. Even though CEA and prolactin have high specificity, their low sensitivity prevents the use of these two markers to differentiate EC and AUB. When AUC was compared for diagnostic significance, CA125 had highest AUC of 0.66.

Kurihara et al. suggested a CA 125 cut-off of $20 \mathrm{U} / \mathrm{ml}$ for predicting myometrial infiltration with sensitivity of $69.0 \%$, specificity of $74.1 \%$, positive predictive value of $58.8 \%$ and negative predictive value of $81.6 \%$. They have also found that elevated CA 125 levels is usually associated with advanced stage and poorer clinical outcome ${ }^{26}$. A study by Kanat-Pektas et al. showed similar diagnostic power for CA 125. The sensitivity, specificity, PPV and NPV for CA 125 in their study was $42.2 \%, 87.4 \%, 77 \%$ and $60.2 \%$ respectively ${ }^{12}$. Among the tumour markers analysed, CA-125 has the comparatively better sensitivity, specificity and high AUC with the cut-off of $17.8 \mathrm{U} / \mathrm{mL}$. Therefore CA-125 can differentiate EC in AUB patients from uterine bleeding due to other causes.

\section{Limitations of the study}

i. The study included small number of patients.

ii. Normal healthy controls were not included since the study was designed to differentiate or diagnose endometrial carcinoma in patients present with AUB and in future study design healthy control group as well as patients with benign uterine diseases will be included.

\section{Conclusion}

We found that serum concentrations of CA 125, CA 15-3, CEA, and Prolactin were significantly higher in endometrial cancer. In addition, we observed that as an individual tumor marker, serum CA 125 has the best diagnostic utility in differentiating endometrial cancer in patients presenting with abnormal uterine bleeding. With reasonable sensitivity and specificity observed in the study, we propose that CA 125 can be best, single and economical test in diagnosing endometrial cancer.

\section{Acknowledgements}

We would like to thank Mr. Vengattaraman, Technical Assistant for his technical support and Mr. Jagannatha P for his guidance in doing statistical analyses.

\section{Grant support}

This study was funded by Jawaharlal Institute of Post graduate Medical Education and Research (JIPMER), Puducherry through intramural grant.

\section{Conflict of interest}

The authors have declared that there is no conflict of Interest.

\section{References}

1. Ferlay J, Soerjomataram I, Dikshit R, Eser S, Mathers C, Rebelo M, et al. Cancer incidence and mortality worldwide: Sources, methods and major patterns in GLOBOCAN 2012: Globocan 2012. Int J Cancer. 2015 Mar 1;1365:E359-86. Epub 2014 Oct 9.

2. Balasubramaniam G, Sushama S, Rasika B, Mahantshetty U. Hospital-based study of endometrial cancer survival in Mumbai, India. Asian Pac J Cancer Prev. 2013;142:97780.

3. Arora V, Quinn MA. Endometrial cancer. Best Pract Res Clin Obstet Gynaecol. 2012 Jun; 263:311-24.

4. Sonoda Y, Barakat RR. Screening and the prevention of gynecologic cancer: Endometrial cancer. Best Pract Res Clin Obstet Gynaecol. 2006 Apr;202:363-77.

5. Sorosky J. Endometrial Cancer: Obstet Gynecol. 2008 Feb;1111:436-47.

6. Amant F, Moerman P, Neven P, Timmerman D, Van Limbergen E, Vergote I. Endometrial cancer. The Lancet. 2005 Aug;3669484:491-505.

7. Yildiz A, Yetimalar H, Kasap B, Aydin C, Tatar S, Soylu F, et al. Preoperative serum CA 125 level in the prediction of the stage of disease in endometrial carcinoma. Eur J Obstet Gynecol Reprod Biol. 2012 Oct;1642:191-5

8. Panici PB, Scambia G, Baiocchi G, Perrone L, Greggi S, Battaglia $F$, et al. Multiple serum markers in patients with endometrial cancer. Gynecol Obstet Invest. 1989;274:208-12. 9. Scambia G, Gadducci A, Panici PB, Foti E, Ferdeghini M, Ferrandina G, et al. Combined use of CA 125 and CA 15-3 in patients with endometrial carcinoma. Gynecol Oncol. 1994 Sep;543:292-7.

10. Neunteufel W, Bieglmayer C, Breitenecker G. CA199, CA125 and CEA in endometrial carcinoma tissue and

African Health Sciences Vol 18 Issue 4, December, 2018 
its relation to hormone receptor content and histological grading. Arch Gynecol Obstet. 1988;2441:47-52.

11. Yurkovetsky Z, Ta'asan S, Skates S, Rand A, Lomakin A, Linkov F, et al. Development of multimarker panel for early detection of endometrial cancer. High diagnostic power of prolactin. Gynecol Oncol. 2007 Oct;1071:58-65.

12. Kanat-Pektas M, Yenicesu O, Gungor T, Bilge U. Predictive power of sexual hormones and tumor markers in endometrial cancer. Arch Gynecol Obstet. 2010 Apr;2814:709-15.

13. Thakur V, Mukherjee U, Kumar K. Elevated serum cancer antigen 125 levels in advanced abdominal tuberculosis. Med Oncol. 2001 Dec;184:289-91.

14. Garrow JS, Webster J. Quetelet's index W/H2 as a measure of fatness. Int J Obes. 1985;92:147-53.

15. Mongia SK, Rawlins ML, Owen WE, Roberts WL. Performance Characteristics of Seven Automated CA 125 Assays. Am J Clin Pathol. 2006 Jun;1256:921-7.

16. Beltran L, Fahie-Wilson MN, McKenna TJ, Kavanagh L, Smith TP. Serum Total Prolactin and Monomeric Prolactin Reference Intervals Determined by Precipitation with Polyethylene Glycol: Evaluation and Validation on Common ImmunoAssay Platforms. Clin Chem. 2008 Oct;5410:1673-81.

17. Lüftner D, Cheli C, Mickelson K, Sampson E, Possinger K. ADVIA Centaur HER-2/neu shows value in monitoring patients with metastatic breast cancer. Int J Biol Markers. 2004 Sep;193:175-82.

18. Erbağci AB, Yilmaz N, Kutlar I. Menstrual cycle dependent variability for serum tumor markers CEA, AFP, CA 19-9, CA 125 and CA 15-3 in healthy women. Dis Markers. 1999 Dec;154:259-67.

19. Kim BW, Jeon YE, Cho H, Nam EJ, Kim SW, Kim
$\mathrm{S}$, et al. Pre-treatment diagnosis of endometrial cancer through a combination of CA125 and multiplication of neutrophil and monocyte. J Obstet Gynaecol Res. 2012 Jan;381:48-56.

20. Bignotti E, Ragnoli M, Zanotti L, Calza S, Falchetti M, Lonardi S, et al. Diagnostic and prognostic impact of serum HE4 detection in endometrial carcinoma patients. Br J Cancer. 2011 Apr 26;1049:1418-25.

21. Jiang T, Huang L, Zhang S. Preoperative serum CA125: a useful marker for surgical management of endometrial cancer. BMC Cancer. 2015 May 12;15:396.

22. Yasa C, Takmaz O, Dural O, Akhan SE. The Value of Tumor Markers in Endometrial Carcinoma: Review of Literature. Journal of Cancer Therapy. 2013 Jun 28;45 2013:966-70.

23. Povolotskaya N, Das N, Dhar K, Brinkmann D, Gardner F, Woolas R. Utility of Preoperative CA125 Assay in the Management Planning of Women Diagnosed with Uterine Cancer. Surg Res Pract. 2014;2014. 497478.

24. Hammarström S. The carcinoembryonic antigen CEA family: structures, suggested functions and expression in normal and malignant tissues. Semin Cancer Biol. 1999 Apr;92:67-81.

25. Kozakiewicz B, Chądzyńska M, Dmoch-Gajzlerska E, Stefaniak M. Monitoring the treatment outcome in endometrial cancer patients by CEA and TATI. Tumour Biol. 2016 Jan 16;377:9367-74.

26. Kurihara T, Mizunuma H, Obara M, Andoh K, Ibuki Y, Nishimura T. Determination of a normal level of serum CA125 in postmenopausal women as a tool for preoperative evaluation and postoperative surveillance of endometrial carcinoma. Gynecol Oncol. 1998 Jun;693:1926. 